\title{
Identifying the Most Accurate Available Space Analysis Method for Predicting Mandibular Third Molar Eruption or Impaction by Means of Panoramic Radiographs
}

\author{
Tahereh Hosseinzadeh Nik, ${ }^{1}$ Toktam Jalayer, ${ }^{2}$ Amine Beymouri, ${ }^{3}$ Atefe Saffar Shahroudi,,${ }^{,}$and Armin \\ Eftekhari $^{3}$ \\ ${ }^{1}$ Professor, Dental Research Center Dentistry Research Institute and Department of Orthodontics, Faculty of Dentistry, Tehran University of Medical Sciences, Tehran, IR Iran \\ ${ }^{2}$ Radiologist, Tehran, IR Iran \\ ${ }^{3}$ Private Practice, Tehran, IR Iran \\ ${ }^{4}$ Department of Orthodontics, Faculty of Dentistry, Lorestan University of Medical Sciences, Khorramabad, IR Iran \\ "Corresponding author: Atefe Saffar Shahroudi, Department of Orthodontics, Faculty of Dentistry, Lorestan University of Medical Sciences, Khorramabad, IR Iran. Tel: \\ +98-9124070738, Fax: +98-44206304, E-mail: a-shahroudi@tums.ac.ir
}

Received 2016 April 21; Accepted 2016 May 06.

\begin{abstract}
Background: Third molar impaction is a major problem facing the dental profession and its impacts on the dentition has long been a major concern. Third molar is the most frequently impacted tooth. The prevalence of mandibular third molar impaction has been reported from 18 to 32 percent which varies between populations and races.

Objectives: This study was designed to determine the best retromolar space analysis from available methods with the highest correlation with mesio-distal width of mandibular third molar (MTM) in order to predict its eruption or impaction.

Patients and Methods: Measurements were carried out on digital panoramic radiographs of 98 Iranian patients. One hundred and seventy mandibular third molars were divided into 4 groups: impacted females, impacted males, erupted females and erupted males. Twenty eight points and 24 planes were marked on the radiographs: 11 points in relation to retromolar space analysis and the mesio-distal width of MTM; and 17 points in relation to some anatomic variables assumed to be related to MTM eruption or impaction. Using these landmarks, different methods were tested for analyzing the retromolar space and its relation to the width of the MTM. Student's t-test was applied for comparison between erupted and impacted groups and ROC curve was drawn to determine the method with the highest specificity and sensitivity.

Results: Among the performed retromolar space analysis, the method that measured the distance between distal surface of second molar and the anterior border of ramus parallel to functional occlusal plane had the highest correlation with the MTM width. Also, the impact of some anatomic factors including posterior ramus height, upper body length, etc. on MTM eruption or impaction was observed.

Conclusions: Retromolar space analysis on panoramic radiographs can be a relatively precise predictor for mandibular third molar eruption or impaction. This study also supports the multifactorial concept about third molar impaction.
\end{abstract}

Keywords: Space Analysis, Mandibular Third Molar, Impaction, Panoramic Radiographs

\section{Background}

Third molar impaction is a major problem facing the dental profession and its impacts on the dentition has long been a major concern. Third molar is the most frequently impacted tooth. The prevalence of mandibular third molar impaction has been reported from 18 to 32 percent which varies between populations and races (1). The average age of MTM eruption has been reported about 20 years ranging from 14 to 24 years $(2,3)$. However, in case of impaction, clinical changes in tooth position have been observed up to 32 years (4).
Impacted and partially erupted teeth can be associated with various problems and pathologic conditions. The etiology of third molar impaction is multifactorial and there is a great uncertainty with the possibility of its eruption. Factors such as insufficient space, genetics, growth direction, the effect of buccinators muscles, external oblique ridge, second molar position, bone resorbtion at the anterior border of ascending ramus, mandibular growth rotation, mesio-distal width of mandibular third molar (MTM) or its angulation and its relative depth has been denoted in the literature (4-6). 
Predicting the impaction or eruption of MTM is important in planning the time of intervention. For example, more complication has been attributed to older age of surgical procedure for MTM removal (whether impacted or partially erupted) (7). Moreover, predicting the risk of impaction can be a considering factor in planning orthodontic treatment plan as reportedly the rate of MTM impaction reduced in patients treated with premolar extraction compared with non-extraction patients $(8,9)$.

Many authors have tried to develop a method for prediction of MTM eruption or impaction However, there is a great uncertainty in these predictions yet. One of the widely accepted explanations for MTM impaction is the discrepancy between the second molar to mandibular ramus space and the mesio-distal width of MTM. Comparing the available space with the mesio-distal width of MTM has been advocated for prediction of MTM impaction. It has been reported that if the mesio-distal available space for MTM is larger than or equal to the mesio-distal width of the tooth, the possibility of eruption is about 70\% (10). Several methods has been applied to measure the retromolar space using panoramic images, lateral chephalograms and CBCT. Although multidetector CT and cone beam CT provides practitioners with the highest quality diagnostic information owing to their dimensionally accurate nature, these imaging methods increase radiation exposure and cost of the procedure and thus are not a part of routine dental examination (11). On the other hand, lateral cephalograms are not prescribed routinely and bilateral analysis would not be accurate or possible in this image modality.

Panoramic images provide a complete overview of the dentition with a relatively low radiation exposure. These radiographs offer a full view of dental arch and associated structures with excellent patient acceptance. The stage of tooth development, the angulation and position of the teeth, and any pathologic condition are observable. Although, there are some innate inaccuracy due to distortion and geometric magnification, the acceptable accuracy of these images and their wide application in routine dental examination has made them potentially appropriate for evaluation and prediction of MTM impaction or eruption $(5,12,13)$. There are several methods for measurement of retromolar space but no comparison to identify the most accurate one was found in our literature review.

\section{Objectives}

The current study was designed to evaluate different recommended methods of retromolar space analysis by the use of panoramic images in adult males and females with and without impaction of mandibular third molar, with the aim of identifying the best method with the highest correlation with the mesio-distal width of the third molar and its eruption or impaction.

\section{Patients and Methods}

This study was carried out on panoramic radiographs of 98 patients who met our inclusion criteria: intact mandibular dental arch, without any missing or extracted tooth, any proximal restoration or crown, presence of bilateral MTM, all patients older than 22 years with evidence of MTM apical root closure, absence of any periapical lesion or extensive caries that affect the pulp of MTM, normal morphology of MTM, no sign of previous trauma on jaws or face, no history of orthodontic or orthognathic surgical treatment. Cases with suboptimal panoramic radiographs were excluded from this study. Digital panoramic radiographs were obtained from a private radiography practice and were taken with a same imaging device (Plamenca Proline XC, Helsinki, Finland).

Totally, 196 MTM were collected from which 26 ones were eliminated due to non-identifiable landmarks in some points; leaving us 170 MTM. The final samples were divided into 4 groups: impacted MTM in females $(\mathrm{N}=52)$, erupted MTM in females $(\mathrm{N}=38)$, impacted MTM in males $(\mathrm{N}=46)$ and erupted MTM in males $(\mathrm{N}=34)$.

The MTM that had the same occlusal level as the adjacent second molar and presented in full occlusion with sufficient space between third molar and ascending ramus was considered as erupted. MTM impaction was defined as incomplete eruption when its normal path of eruption was impeded or blocked by the adjacent tooth. Radiographs were evaluated using tracing papers (14).

Twenty eight points were identified on the radiographs: 11 points in relation to retromolar space analysis and its correlation with the mesio-distal width of the third molar; and 17 points in relation to 16 different anatomic variables that were assumed to be related to third molar eruption or impaction (Figure 1). Using the above 28 points, 24 planes and angles were drawn and measured (Figure 2). All measurements were performed manually using a ruler to the nearest $0.1 \mathrm{~mm}$. To confirm the reliability of measurements, 10 samples were selected randomly and were remeasured. The differences between the two observations were not significant $(\mathrm{P}=0.767)$, indicating the accuracy of the measuring procedure.

In the next step, five different methods were performed to analyze the retromolar space- previously offered by different authors (Table 1). The measured spaces were divided to the width of the MTM to identify the method with the highest correlation. To determine the best retromolar 
space analysis method with regard to the highest sensitivity and specificity, the related ROC curve was drawn in Excel software.

To compare each measure in the two groups of impacted and erupted in males and females and to determine which of the following variables can act as the predictor of the eruption possibility, the independent t-test was applied. The variables comprised: anterior and posterior ramus height, ramus width, upper mandibular body length, first, second and third molar width, the height of retromolar space, anterior and posterior mandibular body width, first and second molar inclination, inner and outer gonial angle and the angle between the central line of the lower one-third of distal root of third molar with the line axis of third molar.

\subsection{Ethics Statement}

The authors warrant this article has been conducted in full accordance with the world medical association declaration of Helsinki. This study was part of an M.S. thesis supported by Dental faculty of Tehran University of Medical Sciences (grant No: 4873-69-02-91) and has been independently reviewed and approved by the ethics committee of this University. There were no patients involved in the study that need patient's permission or any consent form. The radiographs were selected from the archive of a private radiography practice center ant did not have any personal information other than gender and age. The radiographs were taken previously with different diagnostic purposes not for this study.

\section{Results}

From 196 mandibular third molars, 170 were collected and divided into four groups. There were 45 females with 52 impacted and 38 erupted MTM and 40 males with 46 impacted and 34 erupted MTM. The correlations between ten parameters of retromolar space and the width of MTM were calculated. To determine the most precise predictor, the space measured by each of the space analysis parameters was divided to the MTM width. The observed means and standard deviations are demonstrated in Tables 2 and 3. In the impacted groups of males and females, for the first four parameters, the ratio of measured space to the MTM were less than 1, while, in the erupted groups the ratios were more than 1.

A ROC curve was then plotted to identify the method with the highest sensitivity and specificity (Figure 3). According to the plotted curve and Table 4, the A9 method which shows the ratio of the distance between the distal surface of the mandibular second molar and anterior border of ramus parallel to functional occlusal plane to the mesio-distal width of MTM was observed as the most precise method. The A7 method was the next appropriate method followed by A8, A10 and A6 respectively. The specificity and sensitivity for the applied methods in five cuts of points is demonstrated in Tables 5 and 6. Sensitivity stands for the possibility of impaction and specificity shows the possibility of eruption.

The results of measuring sixteen different variables that were assumed to be associated with MTM impaction are summarized in Tables 7 and 8. In the male group, the upper length of mandibular body and the posterior and anterior width of mandibular body were more in patients with erupted MTM than patients with impacted ones. Moreover, posterior ramus height, mesio-distal width of the first, second and third molar, the angulation of second molar and the angle of the lower one-third of third molar's mesial root and long axis of the teeth were less than the impaction group (Table 7).

In the females group, the upper length of mandibular body and the posterior width of body were higher in erupted samples than impacted ones. However, mesiodistal width of the first, second and third molars and the angle of the lower one-third of third molar's mesial root with the long axis of the teeth were less than the impacted samples (Table 8).

\section{Discussion}

In the present study, different retromolar space analyses were evaluated to compare the correlation of the space derived from these methods with the mesio-distal width of the MTM. In the first investigated method in which the distance between the distal surface of second molar to the line tangent to the most concave point on the anterior border of ramus was measured, the mean distance in patients with erupted MTM was 13.6 and $15.3 \mathrm{~mm}$ in females and males respectively. These results meet the Quiroz and Palma suggestion that this distance should be more than $14 \mathrm{~mm}$ (15).

In the next method, the distance between the distal surface of second molar to the anterior aspect of ramus along the functional occlusal plane was measured. The observed data for erupted cases were 14.6 and $16.1 \mathrm{~mm}$ in female and male subjects respectively. The same method which was applied by Hattab et al revealed $14.1 \mathrm{~mm}$ for females and $15 \mathrm{~mm}$ for males. 5 However, in the study that was conducted by Venta et al. to design TME predictor, the average distance in erupted cases was $13.6 \mathrm{~mm}$ which was lower than our results. This discrepancy can be attributed to the racial differences between the two studied populations and also to the small sample size of Venta's study(16). 


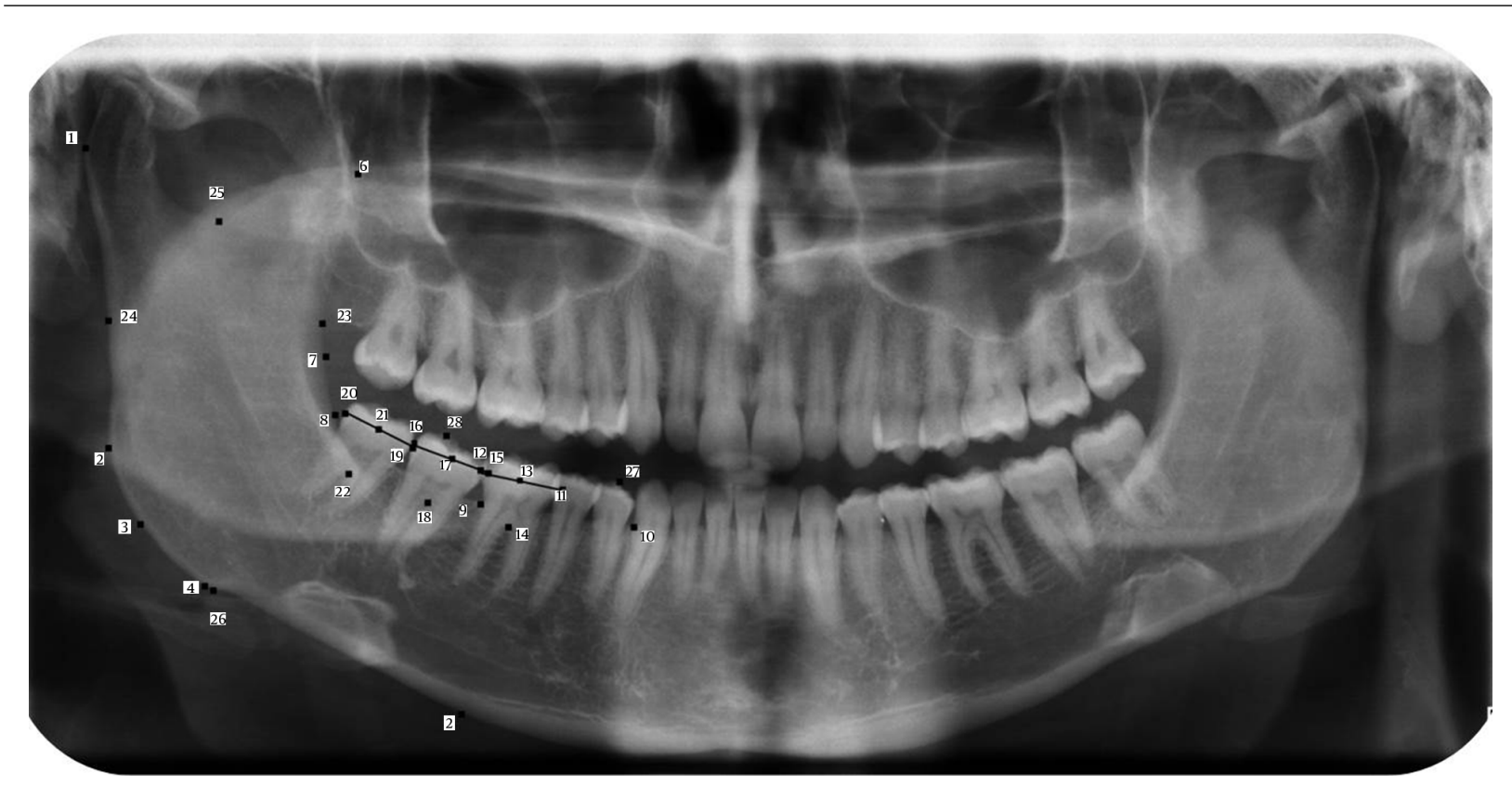

Figure 1. Reference Points Used for Panoramic Radiographs Analysis in the Present Study, 1. A point on condylar head tangent to posterior border of ramus; 2 . A point on mandibular angle tangent to posterior border of ramus; 3 . The outer point on ramus and mandibular body junction; 4 . A point mandibular angle tangent to mandibular plane; 5 . The most inferior point on mandibular border tangent to mandibular plane; 6 . The most anterior superior on coronoid process; 7. The most inferior point on anterior border of ramus; 8 . Interior point ramus mandibular body; 9 . The most superior point on alveolar crest between first and second molar; 10 . The most superior point on alveolar crest between canine and first premolar; 11. The most mesial point on the crown of the first molar; 12. The most distal point on the crown of the first molar; 13. The middle point between the mesial and Distal of the first molar; 14 . A point on the furcation of the first molar root; 15 . The most mesial point on the crown of the second molar; 16. The most distal point on the crown of the second molar; 17. The middle point between the mesial and Distal of the second molar; 18 . A point on the furcation of the first molar root; 19. The most mesial point on the crown of the third molar; 20. The most mesial point on the crown of the third molar; 21 . The middle point between the mesial and Distal of the second molar; 22. A point on the furcation of the first molar root; 23 . The most concave point on the anterior border of ramus; 24 . A point on posterior border of ramus corresponding to the most concave point on the anterior border of ramus; 25 . The deepest point on sigmoid notch on the middle; 26 . A point on the lower border of mandible corresponding to the deepest point on sigmoid notch on the middle; 27 . On the most anterior-superior cusp tip of first premolar; 28 . On the most posterior-superior cusp tip of second molar.

Table 1. Different Methods for Measuring the Retromolar Space (A1 - A5) and the Ratio of the Measured Spaces to Third Molar Width (A6 - A10)

\begin{tabular}{ll}
\hline & Methods \\
\hline A1 & The distance between the distal surface of second molar to the line tangent to the most concave point on the anterior border of ramus \\
A2 & The distance between the distal surface of second molar to the anterior surface of ramus along the functional occlusal plane \\
\hline A3 & The distance between a line perpendicular to the distal surface of second molar to the anterior border of ramus \\
\hline A4 & The distance between the distal surface of second molar to the anterior border of ramus parallel to functional occlusal plane \\
\hline A5 & The distance between the distal surface of second molar to Xi point \\
\hline A6 & The ratio of A1 to the mesio-distal width of third molar \\
A7 & The ratio of A2 to the mesio-distal width of third molar \\
A8 & The ratio of A3 to the mesio-distal width of third molar \\
A9 & The ratio of A4 to the mesio-distal width of third molar \\
A10 & The ratio of A5 to the mesio-distal width of third molar \\
\hline
\end{tabular}

To perform the third method, the distance between a line perpendicular to the distal surface of second molar to the anterior border of ramus was measured. The anterior border of ramus was considered a line from the most superior-anterior convex point of coronoid process to the most inferior concave point on the anterior border of ramus. The average distance in erupted cases was $15 \mathrm{~mm}$ for females and $16.9 \mathrm{~mm}$ for males. There is a relatively large 


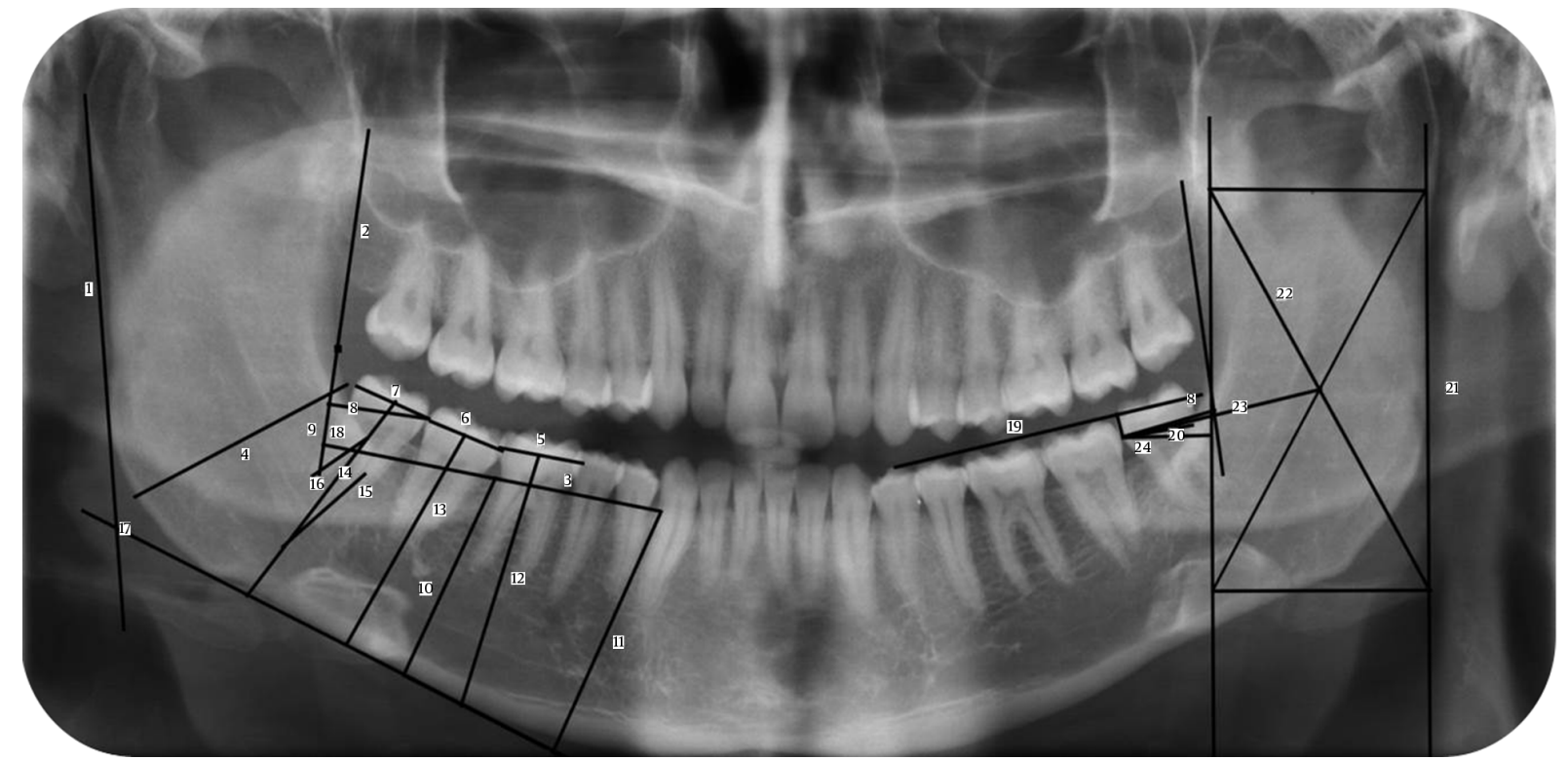

Figure 2. Twenty Four Lines Angle for Estimation of Variables in Impacted and Erupted Third Molars, 1. 1. Posterior ramus height (prh); 2. Anterior ramus height (arh); 3. Upper mandibular body length; 4 . Ramus weight; 5 . First molar width; 6 . second molar width; 7 . Third molar width; 8 . V space (sV): The distance between a perpendicular line from the distal surface of second molar to the anterior border of ramus; 9 . $\mathrm{H}$ space (sH): The distance between junctional point of anterior ramus border and a line which pass along the most superior point of alveolar crest and junctional point of a line perpendicular to the distal surface of second molar to the anterior border of ramus; 10 . Posterior mandibular body width; 11. Anterior mandibular body width; 12. First molar inclination; 13; Second molar inclination; 14; Third molar inclination; 15.The angle between the central line of the lower one-third of mesial root of third molar with the line axis of third molar (tmmra); 16 . The angle between the central line of the lower one-third of distal root of third molar with the line axis of third molar (tmdra); 17. Outer gonial angle (oga): The angle between posterior ramus border and mandibular lower border; 18. Inner gonial angle (iga): The angle between anterior ramus border and the line along with the most superior crestal points; 19 . Functional occlusal plane (fp): A plane that form by connecting anterior-superior cusp tip of first premolar to the most posterior-superior cusp tip of second molar; 20. A plane perpendicular to functional occlusal plane: A line from point 16 to the anterior border of ramus.21.Four lines tangent to points $23,24,25$ and 26 to form ramus square.22. The diameters of ramus square to identify the Xi point; 23. The distance between second molar and Xi; 24 . The distance between the distal surface of second molar to the line tangent to the most concave point on the anterior border of ramus.

Table 2. The Ratio of the Retromolar Space Measured by Each of the Ten Space Analysis Methods to the MTM Width in Females ${ }^{\mathrm{a}}$

\begin{tabular}{|c|c|c|c|c|c|c|c|c|c|c|c|}
\hline \multirow[t]{2}{*}{ Retromolar Space Analysis Methods } & \multicolumn{5}{|c|}{ Impacted } & \multicolumn{6}{|c|}{ Erupted } \\
\hline & $\mathbf{N}$ & Min & Max & Mean & SD & $\mathbf{N}$ & Min & Max & Mean & SD & $\mathbf{P}$ \\
\hline $\begin{array}{l}\text { D-along the tangent line to the most concave point of the } \\
\text { anterior border (A1) }\end{array}$ & 52 & 5.4 & 16.1 & 9.863 & 2.315 & 38 & 9.5 & 19.3 & 13.697 & 2.45 & 0 \\
\hline D-anterior border of ramus along the occlusal plane (A2) & 52 & 6.5 & 17 & 10.273 & 2.232 & 38 & 11.6 & 19.2 & 14.639 & 1.98 & 0 \\
\hline D-along to the constructed anterior border of ramus (A3) & 52 & 7.3 & 18.4 & 11.148 & 2.36 & 38 & 11.8 & 19.3 & 15.068 & 2.269 & 0 \\
\hline D-anterior border of ramus parallel to occlusal plane (A4) & 52 & 3.4 & 14.8 & 8.615 & 2.326 & 38 & 9.8 & 18.4 & 13.097 & 1.888 & 0 \\
\hline D-xi point (A5) & 52 & 20 & 34 & 26.42 & 2.757 & 38 & 25 & 35 & 30.41 & 2.366 & 0 \\
\hline A1/MDW(A6) & 52 & 0.406 & 1.201 & 0.77 & 0.18 & 38 & 0.826 & 1.622 & 1.142 & 0.194 & 0 \\
\hline A2/MDW (A7) & 52 & 0.524 & 1.269 & 0.802 & 0.174 & 38 & 0.935 & 1.637 & 1.222 & 0.164 & 0 \\
\hline A3/MDW (A8) & 52 & 0.566 & 1.373 & 0.871 & 0.188 & 38 & 0.968 & 1.622 & 1.257 & 0.18 & 0 \\
\hline A5/MDW (A10) & 52 & 1.599 & 2.959 & 2.066 & 0.265 & 38 & 2.227 & 3.099 & 2.538 & 0.165 & 0 \\
\hline Valid N (listwise) & 52 & & & & & 38 & & & & & \\
\hline
\end{tabular}

Abbreviations: D, distal surface of mandibular second molar; MDW, mesiodistal width of third molar; SD, standard deviation.

${ }^{\mathrm{a}} \mathrm{P}$ value significant at 0.05 level. 
Table 3. The Ratio of the Retromolar Space Measured by Each of the Ten Space Analysis Parameters to the MTM Width in Males ${ }^{\mathrm{a}}$

\begin{tabular}{|c|c|c|c|c|c|c|c|c|c|c|c|}
\hline \multirow[t]{2}{*}{ Retromolar Space Analysis Methods } & \multicolumn{5}{|c|}{ Impacted } & \multicolumn{6}{|c|}{ Erupted } \\
\hline & $\mathbf{N}$ & Min & Max & Mean & SD & $\mathbf{N}$ & Min & ма & Mean & SD & $\mathbf{P}$ \\
\hline D-along the tangent line to the most concave point of the anterior border (A1) & 46 & 1.7 & 15.5 & 11.172 & 2.843 & 34 & 12 & 19.9 & 15.374 & 2.078 & 0 \\
\hline D-anterior border of ramus along the occlusal plane (A2) & 46 & 1.1 & 15.8 & 11.32 & 2.679 & 34 & 13.1 & 20.1 & 16.144 & 1.97 & 0 \\
\hline D-along to the constructed anterior border of ramus (A3) & 46 & 1.7 & 17 & 12.763 & 2.747 & 34 & 13.4 & 21.2 & 16.9 & 2.082 & 0 \\
\hline D-anterior border of ramus parallel to occlusal plane (A4) & 46 & 0 & 13.5 & 9.3 & 2.712 & 34 & 11 & 17.8 & 14.276 & 1.917 & 0 \\
\hline D-Xi point(A5) & 46 & 21 & 34 & 29.04 & 3.053 & 34 & 28 & 39 & 32.98 & 2.21 & 0 \\
\hline A1/MDW(A6) & 46 & 0.129 & 1.211 & 0.84 & 0.202 & 34 & 1.025 & 1.478 & 1.235 & 0.13 & 0 \\
\hline A2/MDW (A7) & 46 & 0.083 & 1.18 & 0.853 & 0.196 & 34 & 1.073 & 1.595 & 1.299 & 0.137 & 0 \\
\hline A3/MDW (A8) & 46 & 0.129 & 1.3 & 0.962 & 0.201 & 34 & 1.145 & 1.614 & 1.359 & 0.137 & 0 \\
\hline A4/MDW (A9) & 46 & 0 & 0.955 & 0.7 & 0.197 & 34 & 0.887 & 1.413 & 1.149 & 0.136 & 0 \\
\hline A5/MDW A10) & 46 & 1.583 & 2.733 & 2.192 & 0.223 & 34 & 2.398 & 3.255 & 2.658 & 0.161 & 0 \\
\hline Valid N(listwise) & 46 & & & & & 34 & & & & & \\
\hline
\end{tabular}

Abbreviations: D, distal surface of mandibular second molar; MDW, mesiodistal width of third molar; SD, standard deviation

Abbreviations: $D$, distal surface of
a P value significant at 0.05 level.

Table 4. Area Under ROC Curve

\begin{tabular}{lll}
\hline Test Result Variable $(\mathbf{s})$ & Area & \\
\hline A1 & 0.870 & \\
\hline A2 & 0.931 & \\
\hline A3 & 0.877 & 0.941 \\
A4 & 0.832 & 0.946 \\
A5 & 0.980 \\
\hline A6 & 0.951 \\
\hline A7 & 0.987 \\
\hline A8 & 0.947 \\
\hline A9 & \\
\hline A10 & \\
\hline
\end{tabular}

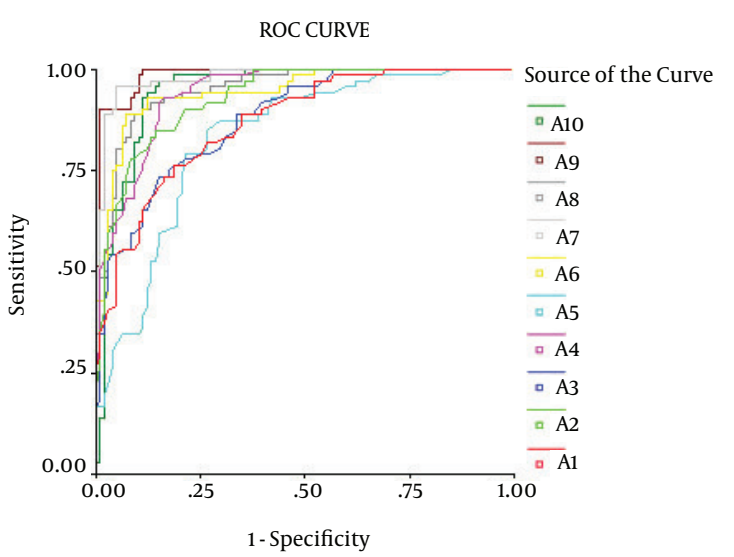

Diagonal Segment Are Produced by Ties.

Figure 3. ROC Curve to Determine the Best Segregation Between Specificity and Sensitivity discrepancy between our results and those of Kaya et al. study (19.1 mm for females and $17.9 \mathrm{~mm}$ for males) in which the retromolar space was wider for females than males (17). This finding was contrary to other studies that reported larger retromolar space for males.

In the fourth method, the distance between the distal surface of second molar to the anterior border of ramus parallel to functional occlusal plane was measured. According to our study, for the MTM to erupt, this space must be more than $9.8 \mathrm{~mm}$ and $11.0 \mathrm{~mm}$ in females and males respectively. The average distance in erupted cases was 13.0 and $14.2 \mathrm{~mm}$ for females and males. Uthman et al. reported $16.0 \mathrm{~mm}$ for females and $16.9 \mathrm{~mm}$ for males (18). However, the authors mentioned a discrepancy between their results and others and attributed the discrepancy to sample selection, racial traits, landmark identification and measuring techniques.

In the current study, it was observed that the distance between the distal surface of second molar to Xi point should be at least $25 \mathrm{~mm}$ in females and $28 \mathrm{~mm}$ in males to make the MTM eruption possible. Turley and Ricketts 
Table 5. The Sensitivity and Specificity A1 to A5 Methods for Retromolar Space Analysis

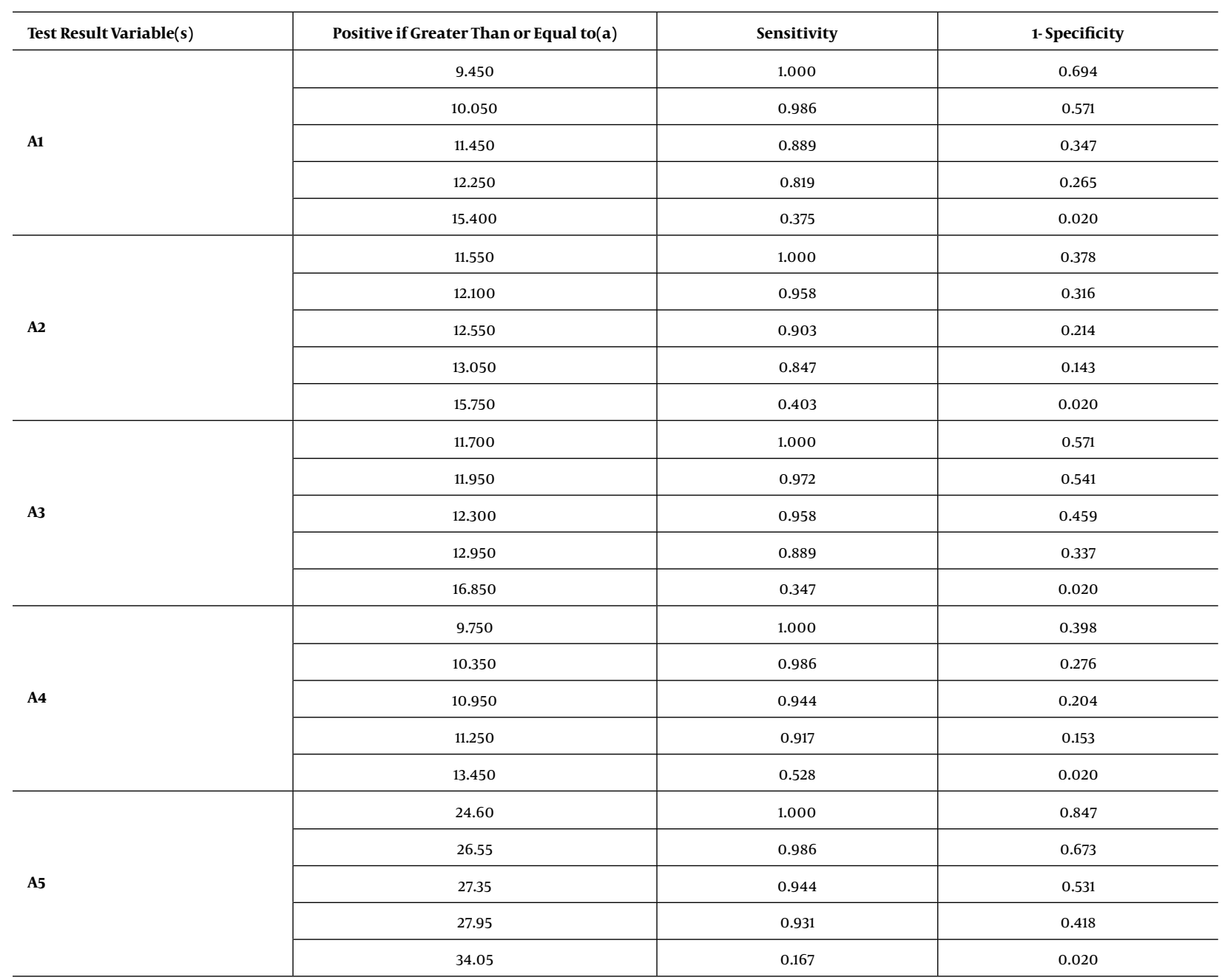

recommended $30 \mathrm{~mm}$ distance for a MTM with appropriate position whereas a minimum space of $35 \mathrm{~mm}$ was suggested by Quiros and Palma (19). Uthman et al. believed that the distance should be more than $27 \mathrm{~mm}$ in females and more than $29 \mathrm{~mm}$ in males (18). The Xi point has been addressed by many authors and the distance between $\mathrm{Xi}$ point and distal surface of second molar has been advocated as the best method for retromolar space analysis (20, 21). Although our results about the distance between $\mathrm{Xi}$ point and second molar was relatively constant with previous reports, this point was not introduced as an appropriate reference due to low specificity and sensitivity of this method and the time consuming process of identifying Xi point.

In the next stage, the ratio of the measured space between each of the above methods to the mesio-distal width of MTM was calculated to evaluate the Ganss et al. ratio (10). In the $\mathrm{A} 6$ method (A1/MDW) $5 \%$ of female and $15 \%$ of males with impacted MTM the ratio of retromolar space to the width of MTM was more than 1. In erupted cases this ratio was more than 1 in all males but in $79 \%$ of females which means that in $21 \%$ of females the MTM erupted although the ratio of retromolar space to MTM width was less than 1. In the A7 method (A2/MDW), 13\% of MTM in females and $23 \%$ in males did not erupt despite a ratio of more than one. This was in accordance with Hattab results in which $13 \%$ of males and $22 \%$ of females with a more than one ratio had MTM impaction (5). Similarly in the A8 method (A3/MDW), $23 \%$ of females and $5 \%$ of males the MTM remained impacted although the ratio was more than one.

It worth to note that in A9 method (A4/MDW) which was the method of choice in this study from specificity and 
Table 6. The Sensitivity and Specificity A1 to A5 Methods for Retromolar Space Analysis

\begin{tabular}{|c|c|c|c|}
\hline Test Result Variable(s) & Positive if Greater Than or Equal to(a) & Sensitivity & 1-Specificity \\
\hline \multirow{5}{*}{ A6 } & 0.82180 & 1.000 & 0.520 \\
\hline & 0.84646 & 0.986 & 0.469 \\
\hline & 0.93840 & 0.944 & 0.255 \\
\hline & 0.98002 & 0.931 & 0.122 \\
\hline & 1.20075 & 0.431 & 0.020 \\
\hline \multirow{5}{*}{ A7 } & 0.93537 & 1.000 & 0.276 \\
\hline & 1.02441 & 0.972 & 0.133 \\
\hline & 1.05086 & 0.958 & 0.051 \\
\hline & 1.09632 & 0.889 & 0.020 \\
\hline & 1.17808 & 0.653 & 0.020 \\
\hline \multirow{5}{*}{ A8 } & 0.96663 & 1.000 & 0.459 \\
\hline & 1.00427 & 0.986 & 0.347 \\
\hline & 1.02415 & 0.958 & 0.276 \\
\hline & 1.07348 & 0.931 & 0.163 \\
\hline & 1.30688 & 0.486 & 0.020 \\
\hline \multirow{5}{*}{ A9 } & 0.88355 & 1.000 & 0.112 \\
\hline & 0.89954 & 0.986 & 0.102 \\
\hline & 0.90660 & 0.944 & 0.092 \\
\hline & 0.95695 & 0.903 & 0.010 \\
\hline & 1.10383 & 0.514 & 0.010 \\
\hline \multirow{5}{*}{ A10 } & 2.22635 & 1.000 & 0.357 \\
\hline & 2.33067 & 0.986 & 0.184 \\
\hline & 2.35166 & 0.972 & 0.153 \\
\hline & 2.44287 & 0.819 & 0.092 \\
\hline & 2.57454 & 0.486 & 0.020 \\
\hline
\end{tabular}

sensitivity stand point, only $2 \%$ of females had impacted MTM despite of a ratio of more than one. In males all MTM erupted when the ratio between the distance between the second molar to anterior ramus border parallel to occlusal plane and the MTM width was more than one.

The fact that in some studies MTM do not erupt in spite of enough retromolar space can be attributed to some inaccuracy in the method of measuring the available space in retromolar area. The results of this study demonstrated that the A9 method can measure the retromolar space with a higher degree of accuracy and higher correlation with the width of MTM. Accordingly, this method can be a better predictor for MTM eruption. However, there were some cases in which the MTM erupted although the ratio of space to tooth width was lesser than one. In order for a MTM to erupt the ratio should be at least 0.9 in females and 0.88 in males.

As mentioned in the previous studies as well as the current one, the retromolar space is the most critical factor in eruption or impaction of MTM. Albeit, it seems some other contributing factor must exist as some times MTM don not erupt in spite of sufficient retromolar space. The angulation of MTM with apical base is one of these factors in the way that the MTM do not erupt when this angle is too low (5). Quiros stated a minimum angle of 40 degree for a MTM to erupt whereas Uthman et al. believed that the angle of the teeth with mandibular plane should be at least between 61 to 65 degree (18).

The width of MTM has been considered as another potential factor. The mean difference between the MTM width in erupted and impacted cases was $0.8 \mathrm{~mm}$ which was statistically significant $(\mathrm{P}=0.01)$. There is considerable con- 
Table 7. The Difference of Sixteen Variables Between Males With Erupted and Impacted Mandibular Third Molar

\begin{tabular}{|c|c|c|c|c|c|c|c|c|c|c|}
\hline \multirow[t]{2}{*}{ Variables } & \multicolumn{4}{|c|}{ Erupted } & \multicolumn{4}{|c|}{ Impacted } & \multirow[t]{2}{*}{ Mean Difference } & \multirow[t]{2}{*}{ PValu } \\
\hline & $\mathbf{N}$ & Mean & SD & SE Mean & $\mathrm{N}$ & Mean & SD & SE Mean & & \\
\hline$r h P$ & 34 & 47.512 & 5.0475 & 0.8656 & 46 & 50.022 & 3.5663 & 0.5258 & -2.51 & $0.011^{\mathrm{a}}$ \\
\hline rhA & 34 & 26.429 & 4.4887 & 0.7698 & 46 & 27.561 & 4.14 & 0.6104 & -1.131 & 0.247 \\
\hline blu & 34 & 54.212 & 3.7029 & 0.635 & 46 & 51.909 & 3.006 & 0.4432 & 2.303 & $0.003^{\mathrm{a}}$ \\
\hline$r w$ & 34 & 38.1 & 3.4257 & 0.5875 & 46 & 38.854 & 3.1613 & 0.4661 & -0.754 & 0.312 \\
\hline$t m w$ & 34 & 12.426 & 0.8203 & 0.1407 & 46 & 13.276 & 0.9884 & 0.1457 & -0.85 & $0^{a}$ \\
\hline smw & 34 & 12.071 & 0.8208 & 0.1408 & 46 & 12.552 & 0.8424 & 0.1242 & -0.482 & $0.013^{\mathrm{a}}$ \\
\hline$f m w$ & 34 & 12.28 & 0.977 & 0.168 & 46 & 12.87 & 0.773 & 0.114 & 0.59 & $0.003^{\mathrm{a}}$ \\
\hline rmsh & 34 & 7.6 & 2.1777 & 0.3735 & 46 & 6.82 & 1.8619 & 0.2745 & 0.78 & 0.089 \\
\hline$b w P$ & 34 & 33.188 & 3.0974 & 0.5312 & 46 & 31.659 & 2.5373 & 0.3741 & 1.53 & $0.018^{\mathrm{a}}$ \\
\hline$b w A$ & 34 & 39.556 & 3.284 & 0.5632 & 46 & 38.107 & 3.0567 & 0.4507 & 1.449 & $0.046^{\mathrm{a}}$ \\
\hline fmi & 34 & 98.28 & 7.719 & 1.324 & 46 & 98.38 & 6.483 & 0.956 & -0.1 & 0.95 \\
\hline$s m i$ & 34 & 89.12 & 6.455 & 1.107 & 46 & 92.05 & 4.667 & 0.688 & -2.94 & $0.021^{\mathrm{a}}$ \\
\hline Go.O & 34 & 117.632 & 6.3045 & 1.0812 & 46 & 117.88 & 6.5304 & 0.9629 & -0.248 & 0.865 \\
\hline Go.I & 34 & 88.44 & 3.886 & 0.666 & 46 & 90.05 & 3.873 & 0.571 & -1.61 & 0.07 \\
\hline Tmmra & 34 & 29.22 & 9.228 & 1.583 & 46 & 35.4 & 13.753 & 2.028 & -6.18 & $0.019^{\mathrm{a}}$ \\
\hline tmdra & 34 & 12.41 & 7.76 & 1.331 & 46 & 14.49 & 11.637 & 1.716 & -2.08 & 0.37 \\
\hline
\end{tabular}

troversy on this issue in the literature as some studies reported a significant difference $(5,6,16)$ while others believed that the difference between the width of erupted and impacted MTM was not significant $(7,18,22)$. This kind of discrepancy can be attributed to the innate inaccuracy of dental panoramic imaging. The objects which are situated buccally are viewed larger than the more lingually situated objects with the same size because of the greater distance from the film.

According to Sewerin investigations, the crown of a MTM is situated buccally in in $65 \%$ of cases and lingually in $4 \%$ of cases and in the middle of the dental arch in the rest of cases (23). To eliminate conventional radiographic errors in the size of third molars, some investigations should be carried out on human skull or extracted and erupted third molars.

In addition to the dimension of third molar, the width of first and second molar should also be taken into account. The determining role of this factor has been advocated by Kaya and Tsai study in which the width of first and second molar was lesser in erupted group than the impacted group $(4,17)$. This is consistent with the observation of the current study.

Gonial angle has also been addressed as a potential contributing factor, albeit in this study as well as some previous investigations $(5,17,18)$. No correlation was observed between this angle and MTM impaction. However, Tsai reported a lower internal gonial angle in erupted group contrary to Behbahani that defined reduced gonial angle as a risk factor of MTM impaction $(4,9)$. The angle between the lower one-third of third molar root with the line axis of third molar (tmdra) can be affected by movements of mandible and teeth especially when the anterior border of the ramus is resorbed and the corpus mandible is enlarged $(24,25)$. Kaya et al. addressed this angle and reported increased risk of impaction with decreased angle particularly the mesial root angle with the tooth line angle (17).

In addition to the aforementioned variables, the upper length of mandibular body and anterior and posterior width of mandibular body was lesser in impaction group. Conversely, posterior ramus height and the angle between second molar and lower mandibular border was more in males with impacted MTM than males with erupted ones.

Regarding the most appropriate age for prediction of MTM eruption or impaction, there is general agreement with other reports that the prediction should be carried out after the age of sixteen. At this age, the normal rotation of MTM crown is relatively completed letting the MTM to keep on the correct course. Moreover, the difference of variables related to position and space of third molar become more pronounced between the two groups of erupted and impacted. Ledyard found out the increase in the space between the second mandibular molar and ramus is less than $2 \mathrm{~mm}$ after the age of 14 and it becomes negligible after the age of 16 . Thus the prediction would be possible after this age.

\subsection{Conclusions}

Within the limitation of this study it was concluded that: 1) The most important contributing factor to the im- 
Table 8. The Difference of Sixteen Variables Between Females With Erupted and Impacted Mandibular Third Molar

\begin{tabular}{|c|c|c|c|c|c|c|c|c|c|c|}
\hline \multirow[t]{2}{*}{ Variables } & \multicolumn{4}{|c|}{ Erupted } & \multicolumn{4}{|c|}{ Impacted } & \multirow[t]{2}{*}{ Mean Difference } & \multirow[t]{2}{*}{ P Value } \\
\hline & $\mathrm{N}$ & Mean & SD & SEMean & $\mathbf{N}$ & Mean & SD & SE Mean & & \\
\hline$r h P$ & 38 & 44.842 & 3.3121 & 0.5373 & 52 & 45.156 & 3.5029 & 0.4858 & -0.314 & 0.669 \\
\hline$r h A$ & 38 & 25.279 & 4.6008 & 0.7463 & 52 & 23.854 & 3.8565 & 0.5348 & 1.425 & 0.114 \\
\hline blu & 38 & 51.858 & 2.2619 & 0.3669 & 52 & 49.725 & 4.3772 & 0.607 & 2.133 & $0.004^{\mathrm{a}}$ \\
\hline$r w$ & 38 & 35.418 & 2.9043 & 0.4711 & 52 & 35.021 & 2.478 & 0.3436 & 0.397 & 0.487 \\
\hline$t m w$ & 38 & 12.011 & 1.0547 & 0.1711 & 52 & 12.867 & 1.1499 & 0.1595 & -0.857 & $0.001^{\mathrm{a}}$ \\
\hline$s m w$ & 38 & 11.979 & 1.0723 & 0.1739 & 52 & 12.675 & 0.9515 & 0.132 & -0.696 & $0.002^{\mathrm{a}}$ \\
\hline fmw & 38 & 12.62 & 1.008 & 0.164 & 52 & 13.13 & 0.979 & 0.136 & -0.51 & $0.018^{\mathrm{a}}$ \\
\hline rmsh & 38 & 6.932 & 2.0344 & 0.33 & 52 & 6.837 & 1.4633 & 0.2029 & 0.095 & 0.797 \\
\hline$b w P$ & 38 & 30.789 & 3.1012 & 0.5031 & 52 & 29.365 & 2.4209 & 0.3357 & 1.424 & $0.016^{\mathrm{a}}$ \\
\hline bwa & 38 & 37.568 & 4.2734 & 0.6932 & 52 & 36.675 & 3.3559 & 0.4654 & 0.893 & 0.288 \\
\hline$f m i$ & 38 & 97.32 & 6.926 & 1.123 & 52 & 98.23 & 5.938 & 0.823 & -0.91 & 0.503 \\
\hline$s m i$ & 38 & 89.03 & 5.739 & 0.931 & 52 & 89.99 & 6.618 & 0.918 & -0.96 & 0.473 \\
\hline Go.O & 38 & 120.618 & 4.9776 & 0.8075 & 52 & 121.798 & 6.4323 & 0.892 & -1.18 & 0.349 \\
\hline Go.l & 38 & 88.68 & 4.945 & 0.802 & 52 & 89.51 & 5.012 & 0.695 & -0.83 & 0.44 \\
\hline Tmmra & 38 & 28.07 & 13.71 & 2.224 & 52 & 40.03 & 18.088 & 2.508 & -11.96 & $0.001^{\mathrm{a}}$ \\
\hline tmdra & 38 & 11.5 & 7.614 & 1.235 & 52 & 16.95 & 12.386 & 1.718 & -5.45 & $0.012^{\mathrm{a}}$ \\
\hline
\end{tabular}

paction or eruption of mandibular third molar is the retromolar space. 2) In estimating the eruption space of third molar, it is recommended to use the ratio of retromolar space to MTM width. The method of choice in this study, was, the distance between the distal surface of second molar to the anterior border of ramus parallel to functional occlusal plane because it serves the highest correlation with the width of MTM with regard to its eruption and had the highest sensitivity and specificity. 3) In the above noted method, the minimum ratio was $0.88 \%$ in erupted males and 0.9 in erupted females. In impacted group, the ratio was $1.1 \%$ and $1.09 \%$ for males and females respectively. 4) The multifactorial etiology of MTM impaction was advocated by the findings of this study. 5) Although the abovementioned factors were slightly different between males and females there were no statistically significant difference in most of variables between males and females with regard to the possibility of third molar impaction.

\section{Acknowledgments}

We would like to appreciate all collogues who help us performing this study and we state that all the authors have viewed and agreed to the submission. It should be mentioned that this study This study was part of a M.S. thesis supported by Tehran University of Medical Sciences (grant No: 4873) and did not receive any other financial support.

\section{Footnotes}

Authors' Contribution: Tahereh Hosseinzadeh Nik participated in the design of the study and its coordination; Toktam Jalayer participated in the design of the study; the radiographs were collected from her office; Amine Beymouri performed the sample preparation and carried out the study measurements; Atefe Saffar Shahroudi prepared the manuscript and she was corresponding author; Armin Eftekhari helped in performing the sample preparation and carrying out the study measurements.

Financial Disclosure: This study was part of a M.S. thesis supported by Tehran University of Medical Sciences (grant No: 4873).

Funding/Support: The authors declare that they did not have any conflict of interest.

\section{References}

1. Andreasen JO. In: Epidemiology of third molar impactions. Andreasen JO, Petersen JK, Laskin DM, editors. ; 1997.

2. Otuyemi OD, Ugboko VI, Ndukwe KC, Adekoya-Sofowora CA. Eruption times of third molars in young rural Nigerians. Int Dent $J$. 1997;47(5):266-70. [PubMed: 9448807].

3. Haralabakis H. Observations on the time of eruption, congenital absence and impaction of the third molar teeth. Trans Eur Orthod Soc. 1957;33(308):9.

4. Tsai HH. Factors associated with mandibular third molar eruption and impaction. J Clin Pediatr Dent. 2005;30(2):109-13. [PubMed: 16491963].

5. Hattab FN, Alhaija ES. Radiographic evaluation of mandibular third molar eruption space. Oral Surg Oral Med Oral Pathol Oral Radiol Endod. 1999;88(3):285-91. [PubMed: 10503855]. 
6. Richardson ME. The etiology and prediction of mandibular third molar impaction. Angle Orthod. 1977;47(3):165-72. doi: 10.1043/00033219(1977)047<0165:TEAPOM>2.0.CO;2. [PubMed: 268949].

7. Mercier P, Precious D. Risks and benefits of removal of impacted third molars. A critical review of the literature. Int J Oral Maxillofac Surg. 1992;21(1):17-27. [PubMed: 1569360].

8. Kim TW, Artun J, Behbehani F, Artese F. Prevalence of third molar impaction in orthodontic patients treated nonextraction and with extraction of 4 premolars. Am J Orthod Dentofacial Orthop. 2003;123(2):138-45. doi:10.1067/mod.2003.13. [PubMed: 12594419].

9. Behbehani F, Artun J, Thalib L. Prediction of mandibular third-molar impaction in adolescent orthodontic patients. Am JOrthod Dentofacial Orthop. 2006;130(1):47-55. doi: 10.1016/j.ajodo.2006.03.002. [PubMed: 16849071].

10. Ganss C, Hochban W, Kielbassa AM, Umstadt HE. Prognosis of third molar eruption. Oral Surg Oral Med Oral Pathol. 1993;76(6):688-93. [PubMed: 8284071].

11. Begtrup A, Gronastoeth HA, Christensen IJ, Kjaer I. Predicting lower third molar eruption on panoramic radiographs after cephalometric comparison of profile and panoramic radiographs. Eur J Orthod. 2013;35(4):460-6. doi: 10.1093/ejo/cjs012. [PubMed: 22474212].

12. Dudhia R, Monsour PA, Savage NW, Wilson RJ. Accuracy of angular measurements and assessment of distortion in the mandibular third molar region on panoramic radiographs. Oral Surg Oral Med Oral Pathol Oral Radiol Endod. 2011;111(4):508-16. doi: 10.1016/j.tripleo.2010.12.005. [PubMed: 21420643].

13. Morris CR, Jerman AC. Panoramic radiographic survey: a study of embedded third molars. J Oral Surg. 1971;29(2):122-5. [PubMed: 5279097]

14. Raghoebar GM, Boering G, Vissink A, Stegenga B. Eruption disturbances of permanent molars: a review. I Oral Pathol Med. 1991;20(4):159-66. [PubMed: 2061854].

15. Quiroz OJ, Palma A. The mandibular third molar: a method for pre- dicting its eruption. Orthodontic Cyber J. 1999.

16. Venta I, Murtomaa H, Ylipaavalniemi P. A device to predict lower third molar eruption. Oral Surg Oral Med Oral Pathol Oral Radiol Endod. 1997;84(6):598-603. [PubMed: 9431525].

17. Kaya G, Aslan M, Omezli M, Dayi E. Some morphological features related to mandibular third molar impaction. J Clin Exp Dent. 2010;2(1):12-7.

18. Uthman AT. Retromolar space analysis in relation to selected linear and angular measurements for an Iraqi sample. Oral Surg Oral Med Oral Pathol Oral Radiol Endod. 2007;104(4):e76-82. doi: 10.1016/j.tripleo.2007.05.013. [PubMed: 17703969].

19. Ricketts RM, Turley P, Chaconas S, Schulhof RJ. Third molar enucleation: diagnosis and technique. J Calif Dent Assoc. 1976;4(4):52-7. [PubMed: 1074850].

20. Schulhof RJ. Third molars and orthodontic diagnosis. J Clin Orthod. 1976;10(4):272-81. [PubMed: 1074882].

21. Qamruddin I, Qayyum W, Haider SM, Siddiqui SW, Rehan F. Differences in various measurements on panoramic radiograph among erupted and impacted lower third molar groups. J Pak Med Assoc. 2012;62(9):883-7. [PubMed: 23139968]

22. Richardson ER, Malhotra SK, Semenya K. Longitudinal study of three views of mandibular third molar eruption in males. Am J Orthod. 1984;86(2):119-29. [PubMed: 6589957].

23. Sewerin I. Preoperative radiographic examination of the mandibular 3rd molars using 4 projections. Tandlaegebladet. 1984;88(1):1.

24. Yamaoka M, Furusawa K, Hayama H, Kura T. Relationship of third molar development and root angulation. J Oral Rehabil. 2001;28(2):198205. [PubMed: 11298270].

25. Marklund M, Persson M. The relationship between mandibular morphology and apical root curvature in man. Arch Oral Biol. 1988;33(6):391-4. [PubMed: 3228384]. 